\title{
Modelling uncertainty in stochastic multicriteria acceptability analysis
}

\author{
Ian N. Durbach ${ }^{* 1,2}$ and Jon M. Calder ${ }^{1}$ \\ ${ }^{1}$ Department of Statistical Sciences, University of Cape Town, Rondebosch 7701, \\ South Africa \\ ${ }^{2}$ African Institute for Mathematical Sciences, 6-8 Melrose Road, Muizenberg 7945, \\ South Africa
}

\begin{abstract}
This paper considers problem contexts in which decision makers are unable or unwilling to assess trade-off information precisely. A simulation experiment is used to assess (a) how closely a rank order of alternatives based on partial information and stochastic multicriteria acceptability analysis (SMAA) can approximate results obtained using full-information multi-attribute utility theory (MAUT) with multiplicative utility, and (b) which characteristics of the decision problem influence the accuracy of this approximation. We find that fairly good accuracy can be achieved with limited preference information, and is highest if either quantiles and probability distributions are used to represent uncertainty.
\end{abstract}

\section{Introduction}

When facilitating decisions it sometimes happens that some inputs to the preference model either cannot be assessed at all or can only be assessed within relatively large bounds of uncertainty (e.g. Jessop, 2014; Ahn and Park, 2014; Kadziński et al., 2015). This can happen for a number of reasons: a lack of time, a politically sensitive problem context, or a lack of decision maker (DM) involvement, for example. Whatever the reason, in these cases the DM is unable or unwilling to express him or herself with the degree of precision required by conventional decision aids. We call decision problems which must be addressed under such conditions "low-involvement" decisions. The question is what, if any, decision support can be provided in such situations.

Stochastic multicriteria acceptability analysis (SMAA, Tervonen and Figueira (2008); Lahdelma and Salminen (2012)) is a family of decision models that can be used with arbitrarily precise preference information. It addresses low-involvment decision-making

\footnotetext{
${ }^{*}$ Corresponding author: ian.durbach@uct.ac.za
} 
by providing information about the types of preferences (if any) that would lead to the selection of each alternative.

In this paper we use a simulation experiment to evaluate the ability of SMAA to approximate results obtained using multi-attribute utility theory (MAUT) where preferences are represented by a multiplicative utility function. In particular, we ask how closely results computed from a key output from SMAA (the acceptability index) can approximate those obtained using MAUT. In doing so we hope to provide a broad indication of the losses that are possible if facilitators choose to use a low-involvement decision aid such as SMAA rather than compelling DMs to be more precise in their assessment of certain types of preference information - for example, using more detailed problem structuring. We also wish to test the robustness of the SMAA approach to various aspects of the decision process: the size of the decision problem, the way attribute evaluations are distributed, the underlying preference functions, the accuracy of assessed information, the amount of preference information gathered, and the way in which the acceptability index is constructed.

In addition to the conventional SMAA model, which uses probability distributions to represent uncertainty in the attribute evaluations, we also introduce and evaluate a number of 'simplified' models which make use of summarized measures of uncertainty instead of a full probability distribution (Durbach and Davis, 2013). By assessing the accuracy of both conventional and simplified uncertainty models under a range of different conditions we hope to provide motivation for the use of simplified models in appropriate circumstances. A similar approach has been used in Durbach and Stewart (2012) to assess the effect of using simplified uncertainty formats in general decision-making, and we employ a similar simulation structure in the current paper.

Our view is that in nearly all cases it is preferable to resolve preferential uncertainty through discussion and problem structuring rather than by employing more 'lenient' decision aids, because of the additional insight and opportunities for learning. We focus on those circumstances in which the DM is unable or unwilling to participate fully in this process. In using a simulation experiment, we acknowledge that we can only evaluate the extent to which using SMAA rather than MAUT might impact results. We cannot evaluate critical issues like whether the reduced time spent on problem structuring in SMAA is "worth" the reduction in decision quality, or the degree to which the problem structuring process, through the insight it generates for the DM, is useful as an end in itself. Simula- 
tion results are unable to provide general conclusions on the viability of different methods, but provide inputs to such discussion by identifying the potential trade-offs in accuracy that are implied when using a simplified model. Ultimately accuracy must be weighed against other factors to determine which decision model may be most appropriate for a problem.

The remainder of the paper is structured as follows. Section 2 provides a review of the relevant background literature and notation. Section 3 describes the structure of our simulation experiment. Section 4 presents the results in a direct fashion, delaying a more detailed discussion of these until Section 5, which also provides theoretical justification for some key findings using results from applied probability theory. Section 6 discusses implications of the simulation results for the use of SMAA as a prescriptive decision aid, and concludes.

\section{Notation and background}

Consider a decision problem consisting of $I$ alternatives $\left\{a_{1}, a_{2}, \ldots, a_{I}\right\}$ evaluated on $J$ attributes $\left\{c_{1}, c_{2}, \ldots, c_{J}\right\}$. Let $Z_{i j}$ be a random variable denoting the attribute evaluation of $a_{i}$ on $c_{j}$, and $U$ be a multi-attribute utility function mapping the attribute evaluations of alternative $a_{i}$ (denoted $\mathbf{Z}_{i}$ ) to a real value using a weight vector $\mathbf{w}$. A joint density function $f_{X}(\mathbf{Z})$ governs the generation of the $Z_{i j}$ in the space $X \subseteq R^{I \times J}$, and a second joint density function $g(\mathbf{w})$ governs the generation of imprecise or unknown weights in the weight space $W$. Total lack of knowledge is usually represented by a uniform distribution in $W$. If restrictions have been placed on $W$ we denote the feasible weight space by $W^{\prime}$.

The original SMAA method (Lahdelma et al., 1998) analysed the combinations of attribute weights that result in each of a set of alternatives being selected when using an additive utility function. Subsequently, a number of SMAA variants have been developed. These differ in terms of the preference model used and thus the type of preference information that is imprecisely known, but are all based upon Monte Carlo simulation from distributions which govern unknown preference parameters (and attribute evaluations). For example, SMAA variants are available for value function (Lahdelma et al., 1998; Lahdelma and Salminen, 2001), outranking (Hokkanen et al., 1998), reference point (Lahdelma et al., 2005; Durbach, 2006), prospect theory, (Lahdelma and Salminen, 2008), Choquet integral (Angilella et al., 2015), and AHP (Durbach et al., 2014) methods. Comprehensive reviews are given by Tervonen and Figueira (2008) and Lahdelma and Salminen 
(2012).

Given a particular weight vector w, the global utility of each alternative can be computed and a rank ordering of alternatives obtained. SMAA-2 (Lahdelma and Salminen, 2001 ) is based on simulating a large number of random weight vectors from $g(\mathbf{w})$ and observing the proportion and distinguishing features of weight vectors which result in each alternative obtaining a particular rank $r$ (usually the "best" rank, $r=1$ ), using an additive value function model. Let the set of weight vectors that result in alternative $a_{i}$ obtaining rank $r$ be denoted by $W_{i}^{r}$. SMAA is based on an analysis of these sets of weights using a number of descriptive measures, the most important of which are:

Acceptability indices The rank- $r$ acceptability index $b_{i}^{r}$ measures the proportion of all simulation runs i.e. weight vectors, that make alternative $a_{i}$ obtain rank $r$. A cumulative form of the acceptability index called the $R$-best ranks acceptability index is defined as $\mathcal{B}_{i}^{R}=\sum_{r=1}^{R} b_{i}^{r}$ and measures the proportion of all weight vectors for which alternative $a_{i}$ appears anywhere in the best $R$ ranks. In the discussion in Section 5 we make use of ordered acceptability indices, where we denote the alternative with the $k$-th largest rank- $r$ acceptability index by $a_{(k)}^{r}$, and its acceptability index by $b_{(k)}^{r}$.

Central weight vectors The central weight vector $\mathbf{w}_{i}^{c}$ is defined as the expected center of gravity of the favourable weight space $W_{i}^{1}$. It gives a concise description of the "typical" preferences supporting the selection of a particular alternative $a_{i}$, and in practice is computed from the empirical (element-wise) averages of all weight vectors supporting the selection of $a_{i}$ as the best alternative.

The exact number of Monte Carlo iterations that are required to achieve a given accuracy is discussed in Tervonen and Lahdelma (2007). To estimate the acceptability index within $\delta$ of the true value with $95 \%$ confidence, one requires $1.96^{2} / 4 \delta^{2}$ iterations so that 10000 iterations will usually be sufficient to achieve error bounds of $1 \%$.

Uncertain attribute evaluations are conventionally treated in SMAA using probability distributions, with each simulation run drawing values at random from these distributions. Adapting SMAA to use other uncertainty formats, however, is generally straightforward, as described in Durbach and Davis (2013). Each uncertain attribute is simply replaced by a number of lower-level attributes which capture the uncertainty in the evaluations on that attribute, using one of many possible simplified uncertainty formats. In this paper, we test 
the effect of using three different formats: expected values; variances; and quantiles. This transforms the decision problem into one having the same appearance as a deterministic decision problem, which can be treated by any of the existing SMAA models with some minor modifications. We sometimes refer to these collectively as "SMAA models" although it should be clear that these are approximations of external uncertainty built on top of the same SMAA approach. The models are described in more detail in Section 3. Table 1 provides a summary of notation used in the paper.

\section{Description of the simulation study}

The general structure of the simulation ${ }^{1}$ is summarized in Figure 1 and implements following basic steps:

1. Form a hypothetical problem context, generating the relevant attribute evaluations.

2. Apply a multiplicative MAUT model to derive idealised or 'true' utilities and thus find the 'true' rank ordering of alternatives.

3. Calculate summarized measures of uncertainty based on the generated data and incorporating observational errors.

4. Run different models using SMAA and the inputs from step 3, and then compare the model results against the 'true' utilities and rank order obtained from step 2.

\subsection{Generating attribute evaluations}

We consider a decision problem involving $I$ alternatives evaluated over $J$ attributes. External uncertainty about attribute performance is captured by simulating attribute evaluations for alternative $a_{i}$ on attribute $c_{j}$ from a gamma distribution with mean $\mu_{i j}$, standard deviation $\sigma_{i j}$, and skewness $\xi_{i j}$. We denote this distribution $f_{i j}\left(Z_{i j}\right)$. Each mean $\mu_{i j}$ is drawn randomly from a uniform distribution between 0.2 and 0.8 for all alternatives on all attributes. Means are then standardized across all the attributes to lie on the unit hypersphere i.e. $\sum_{j=1}^{J} \mu_{i j}^{2}=1, \forall i$. This creates a set of non-dominated alternatives, at least in this 'average' sense. The standard deviations and skewnesses of the attribute evaluations are varied as parameters of the simulation (see Section 3.6 for details of chosen parameters values). A set of $K=15000$ realizations is generated to represent the uncertain attribute

\footnotetext{
${ }^{1}$ All code used to run the simulations described in this section is openly available from http://dx.doi.org/10.5281/zenodo. 30523.
} 


\section{Problem context}

$a_{i}$

$c_{j}$

$c_{(k)}$

$Z_{i j}$

$f_{X}(\mathbf{Z})$

$f_{i j}\left(Z_{i j}\right)$ or $f_{i j}$ alternative $i$

attribute $j$

attribute with $k$-th largest importance weight

performance of $a_{i}$ on $c_{j}$

joint probability distribution over all $Z_{i j}$

marginal probability distribution for $Z_{i j}$

\section{Attribute weights}

$W$

$W^{\prime}$

$W_{i}^{1}$

$w_{j}$

$w_{(1)}$

SMAA

$b_{i}^{r}$

$b_{(k)}^{r}$

$a_{(k)}^{r}$

$B_{(k)}^{1}$

$\mathcal{B}_{i}^{R}$ total weight space

currently feasible weight space

favourable weight space for $a_{i}$

weight for $c_{j}$

$k$-th largest attribute importance weight

rank- $r$ acceptability index

$k$-th largest rank- $r$ acceptability index

alternative with $k$-th largest rank- $r$ acceptability index

random variable which when realized gives $b_{(k)}^{1}$

cumulative $R$-best ranks acceptability index

\section{Simulation: problem context}

I

$J$

K

$z_{i j k}$

$\mu_{i j}$

$\sigma_{i j}$

$\xi_{i j}$

number of alternatives

number of attributes

number of simulated realizations of $Z_{i j}$

realization $k$ of $Z_{i j}$

mean of attribute evaluations for $a_{i}$ on $c_{j}$

standard deviation of attribute evaluations for $a_{i}$ on $c_{j}$

skewness of attribute evaluations for $a_{i}$ on $c_{j}$

\section{Simulation: DM preferences}

$v_{j}$

$\tau_{j}$

$\lambda_{j}$

$V_{i}$

$U_{i}$

$\zeta$

$\omega$

marginal value function for $c_{j}$

reference level for $v_{j}$

value of $v_{j}$ at reference level

global value of $a_{i}$

global utility of $a_{i}$

risk preferences defined over value

'true' attribute weights

\section{Simulation: uncertainty modelling}

$\rho \quad$ proportion of importance ranks provided by DM

$\epsilon$

$z_{i j}^{\prime}$

size of assessment errors

sampled value from $z_{i j}$. used by 'TDist' model

sampled value from $f_{i j}$ used by 'EDist' model

$E\left[Z_{i j}\right], \hat{E}\left[Z_{i j}\right]$

expected value of $Z_{i j}$ before and after application of $\epsilon$

$\operatorname{VAR}\left[Z_{i j}\right], \operatorname{VAR}\left[Z_{i j}\right] \quad$ variance of $Z_{i j}$ before and after application of $\epsilon$

$Q_{r}\left[Z_{i j}\right], \hat{Q}_{r}\left(Z_{i j}\right) \quad 5 \%, 50 \%$ and $95 \%$ quantiles of $Z_{i j}$ for $r=1,2,3$ before and after application of $\epsilon$ weight of $\operatorname{VAR}\left[Z_{i j}\right]$ relative to $E\left[Z_{i j}\right]$

Table 1: Summary of all parameters used 
Generate 'true' preference information

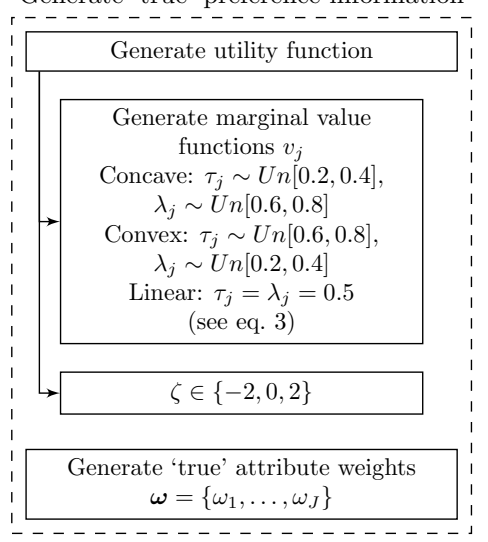

Generate attribute realizations, given problem context parameters $I, J$, var, skew

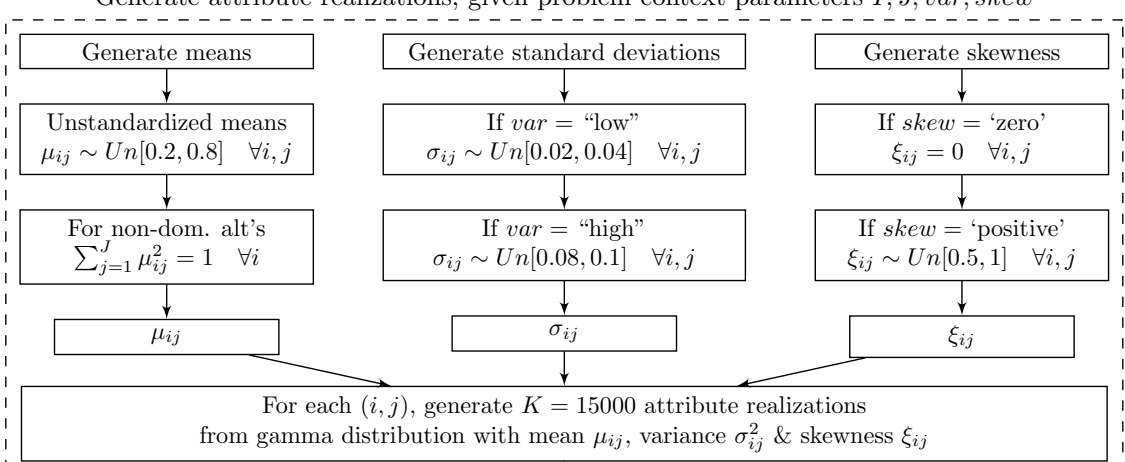

from gamma distribution with mean $\mu_{i j}$, variance $\sigma_{i j}^{2} \&$ skewness $\xi_{i j}$

Standardise realizations within each attribute $c_{j}$ to lie between 0 and 1 (over all alternatives) Denote $z_{i j k}$

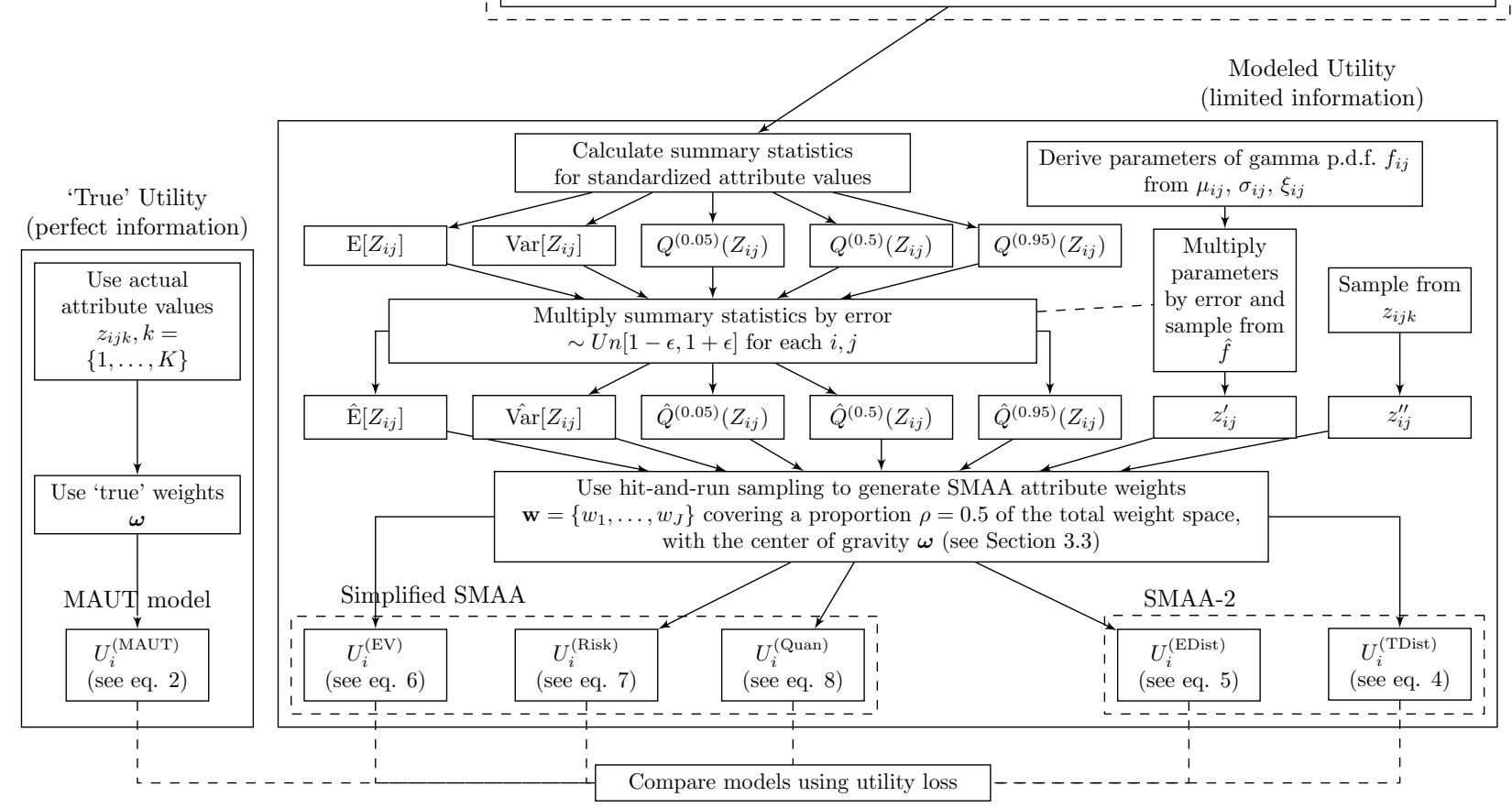

Figure 1: Outline of a simulation run

evaluation of each alternative on each attribute. These evaluations, denoted $z_{i j k}$, are then standardized within each attribute (across all alternatives and realizations), to have a minimum of 0 and a maximum of 1 .

\subsection{Generating preference structures}

We employ a multiplicative MAUT model to simulate the 'true' underlying preference structure of a DM, with $U_{i}$ denoting the overall utility of alternative $a_{i}$. For simplicity we assume our simulated DM has a utility function that exhibits constant risk preferences over the scalar value attribute $V_{i}$. Specifically, this requires that the DM's preferences over the attribute space are compatible with an additive value function, that at least one 
attribute is utility independent of its complement, and that $J \geq 3$ (Keeney and Raiffa, 1976, Theorem 6.11). These conditions allow us to rewrite the global utility of alternative $a_{i}$ as a function of its global value $V_{i}$, and more precisely as a function of its value as derived from an additive value model:

$$
U_{i}= \begin{cases}e^{-\zeta V_{i}}=\exp \left[-\zeta \sum_{j=1}^{J} w_{j} v_{j}\left(Z_{i j}\right)\right] & \zeta<0 \\ V_{i}=\sum_{j=1}^{J} w_{j} v_{j}\left(Z_{i j}\right) & \zeta=0 \\ -e^{-\zeta V_{i}}=-\exp \left[-\zeta \sum_{j=1}^{J} w_{j} v_{j}\left(Z_{i j}\right)\right] & \zeta>0\end{cases}
$$

where $w_{j}$ denotes the relative importance weight of attribute $c_{j}, v_{j}$ is the marginal value function for $c_{j}$, and $\zeta$ determines whether the DM is constant risk averse $(\zeta>0)$, risk neutral $(\zeta=0)$ or constant risk prone $(\zeta<0)$ with respect to value. The precise form of the marginal value function $v_{j}$ is discussed in Section 3.2.1. To calculate the 'true' utility of an alternative $a_{i}$, expectations are taken over the full set of $K$ realisations. That is, we (a) compute, for each $k$, the value $V_{i k}$ and hence utility $U_{i k}$ of alternative $a_{i}$ using attribute evaluations $z_{i 1 k}, \ldots, z_{i J k}$; (b) average these utilities over $k$. This gives

$$
U_{i}^{\mathrm{MAUT}}= \begin{cases}E\left[e^{-\zeta V_{i}}\right]=(1 / K) \sum_{k=1}^{K} \exp \left[-\zeta \sum_{j=1}^{J} w_{j} v_{j}\left(z_{i j k}\right)\right] & \zeta<0 \\ E\left[V_{i}\right]=(1 / K) \sum_{k=1}^{K} \sum_{j=1}^{J} w_{j} v_{j}\left(z_{i j k}\right) & \zeta=0 \\ -E\left[e^{-\zeta V_{i}}\right]=(1 / K) \sum_{k=1}^{K}-\exp \left[-\zeta \sum_{j=1}^{J} w_{j} v_{j}\left(z_{i j k}\right)\right] & \zeta>0\end{cases}
$$

This 'true' measure of utility is based on perfect and complete information and, for the purposes of this simulation study, provides a set of idealised results to which results from the different SMAA models can be compared.

\subsubsection{Marginal value functions}

Marginal value functions are assumed to be piecewise linear between the points $(0,0)$, $\left(\tau_{j}, \lambda_{j}\right)$, and $(1,1)$. Convex, linear, and concave value functions can be generated by imposing $\tau_{j}>\lambda_{j}, \tau_{j}=\lambda_{j}$, and $\tau_{j}<\lambda_{j}$ respectively, allowing a wide range of preference to be simulated parsimoniously. Note that this is a simplified version of the approach used in Durbach and Stewart (2012), where additional parameters were used to control the degree of curvature in the preference functions either side of $\tau_{j}$. Marginal values are given by:

$$
v_{j}(x)= \begin{cases}\frac{\lambda_{j} x}{\tau_{j}} & \text { for } 0 \leq x \leq \tau_{j} \\ \lambda_{j}+\frac{1-\lambda_{j}}{1-\tau_{j}}\left(x-\tau_{j}\right) & \text { for } \tau_{j}<x \leq 1\end{cases}
$$




\subsubsection{Inter-attribute weights}

'True' attribute weights $\boldsymbol{\omega}$ are simulated to be uniformly distributed and sum to one, using the approach in Butler et al. (1997). This involves sorting $J-1$ randomly generated $U n[0,1]$ variates in ascending order, appending 0 and 1 to the extremes of this sequence, and extracting the differences between adjacent values.

\subsection{Simulating the application of SMAA}

The 'true' attribute weights $\boldsymbol{\omega}$ provide a rank ordering of attributes from most important to least important. Let $c_{(k)}$ denote the $k$-th most important attribute. To emulate the inclusion of partial preference information in the SMAA models, we simulate a DM who provides the importance ranks of a proportion $\rho$ of the full set of attributes (less one, since the rank of the final attribute is given by the others), in either decreasing (starting from $c_{(1)}$ ) or increasing (starting from $c_{(J)}$ ) order of importance. Total uncertainty regarding weights corresponds to $\rho=0$ while for a complete rank ordering $\rho=1$.

Efficient "hit-and-run" sampling procedures using Markov Chain Monte Carlo (MCMC) methods to simulate uniformly distributed weights on a simplex with arbitrary constraints have been described in Tervonen et al. (2013) and van Valkenhoef et al. (2014). Importance ranks provide ordinal information that is easily implemented in this framework as linear constraints on the weight space e.g. $w_{(1)}>w_{(k)}, \forall k \neq 1$. The hit-and-run procedure is implemented in $\mathrm{R}$ using the 'hitandrun' package (van Valkenhoef and Tervonen, 2015). The computational costs of the simulation are already fairly high, so that $\rho$ is not included as one of the final simulation parameters but is rather the subject of an initial investigation before being set at a fixed value of 0.5 (see Section 4.1 for details).

Finally, our SMAA models assume that marginal value functions are known and assessed without error, but that the parameter $\zeta$ governing risk preferences defined over value is unknown. Although in principle it is straightforward to simulate possible values of $\zeta$, or parameters of marginal value functions, as part of each SMAA model, assuming known value functions but unknown weights is, perhaps regrettably, a common feature of SMAA, which we follow here to prevent the simulations from becoming overly complex. We thus assume that low-involvement models like SMAA may well assume $\zeta=0$, approximating the multiplicative utility function with an additive value function and ranking alternatives according to their global values as returned by the respective SMAA models described below. 


\subsubsection{Model using a full probability distribution}

We implement two SMAA models employing full probability distributions. The first randomly samples, at each SMAA iteration, values from the empirical distribution of the standardized attribute values, and thus directly samples from the $z_{i j k}$. The resulting "true distribution" model is given by

$$
U_{i}^{\text {(TDist) }}=\sum_{j=1}^{J} w_{j} v_{j}\left(z_{i j}^{\prime}\right)
$$

where $z_{i j}^{\prime}$ is the sampled value from $z_{i j}$. Note that this model captures uncertainty in the attribute evaluations exactly, by construction, but is not easily amenable to modelling assessment errors. We therefore also use a second distributional model that randomly draws attribute evalutions from the $f_{i j}\left(Z_{i j}\right)$ at each SMAA iteration. Assessment errors can be modelled in a straightforward way by imposing errors on the parameters of $f_{i j}$. The "estimated distribution" model is given by

$$
U_{i}^{(\mathrm{EDist})}=\sum_{j=1}^{J} w_{j} v_{j}\left(z_{i j}^{\prime \prime}\right)
$$

where $z_{i j}^{\prime \prime}$ refers to the sampled attribute evaluations, now understood to be drawn from the $f_{i j}\left(Z_{i j}\right)$ rather than the empirical distributions.

\subsubsection{Model using expected values}

The model using only expected values ignores any uncertainty in the attributes and is defined as:

$$
U_{i}^{(\mathrm{EV})}=\sum_{j=1}^{J} w_{j} v_{j}\left(E\left[Z_{i j}\right]\right)
$$

Expected value models effectively reverse the order of expectation and utility operations. Of course if expectations can only be estimated from the full distributions then (6) is of little use, but it may be the case that the DM is able to assess an average fairly easily, without reference to the full distribution. In these cases (6) may be thought of as a "simplified" approach. The model is investigated in Kirkwood (1992); Stewart (1996), and Durbach and Stewart (2009). 


\subsubsection{Model using expected values and variances}

This model uses variances in addition to expected values and is referred to here as a "risk" model. It is defined as:

$$
U_{i}^{(\mathrm{Risk})}=\sum_{j=1}^{J} w_{j} v_{j}\left(E\left[Z_{i j}\right]\right)-\phi \sum_{j=1}^{J} w_{j} \operatorname{VAR}\left[Z_{i j}\right]
$$

where $\phi$ is a multiplier indicating the contribution of the risk component relative to the expected value component. Here we set $\phi=0.25$, which is smaller than the values used in Durbach and Stewart (2012) but follows results in that paper reporting poor performance with larger values of $\phi$.

\subsubsection{Model using quantiles}

The quantile model makes use of the "extended Pearson-Tukey" approximation (Keefer and Bodily, 1983), a widely-applicable three-point approximation for continuous probability distributions typical of those elicited via judgmental assessments.

$$
U_{i}^{(\text {Quan })}=\sum_{j=1}^{J} w_{j} \sum_{r=1}^{3} x_{r} v_{j}\left(Q_{r}\left[Z_{i j}\right]\right)
$$

with $x_{1}=x_{3}=0.185$ and $x_{2}=0.63$ and where $Q_{1}, Q_{2}$, and $Q_{3}$ are the $5 \%, 50 \%$ and $95 \%$ quantiles respectively.

\subsection{Generating errors in the assessment of uncertainty information}

In order to allow for assessment errors, all model inputs relating to uncertainty are multiplied by randomly generated realizations from $U n[1-\epsilon, 1+\epsilon]$. The error factor $\epsilon$ is a parameter of the simulation - we use $0 \%, 10 \%$ and $20 \%$ errors. The error adjusted parameter values are then denoted using the 'hat' symbol i.e. assessed probability distributions $\hat{f}\left(Z_{i j}\right)$, expected values $\hat{E}\left[Z_{i j}\right]$, variances $\operatorname{VÂR}\left[Z_{i j}\right]$, and quantile values $\hat{Q}_{r}\left(Z_{i j}\right)$. Errors are not applied to the "true distribution" model sampling directly from the $z_{i j k}$. In the case of the "estimated distribution" model errors are applied to both shape and scale parameters of the assumed gamma distributions governing attribute evaluations before renormalizing attribute evaluations to lie between zero and one.

\subsection{Output Measures}

For each SMAA model, alternatives are ranked by their $R$-best ranks acceptability indices, where $R \in\{1,3,5\}$ is varied as a parameter of the simulation. To evaluate the quality 
of the alternatives selected by each SMAA model we use three outcomes: the probability that the alternative selected by MAUT appears in the top one, three, or five positions of the SMAA rank order. The first of these tests the ability of SMAA with respect to the choice problematique, the others with respect to the shortlisting of promising alternatives.

In previous simulations (e.g. Durbach and Stewart, 2009, 2012) we have primarily used 'utility loss' $U L=\left(U_{i^{*}}-U_{i^{s e l}}\right) /\left(U_{i^{*}}-U_{i_{*}}\right)$ to measure model accuracy, where $U_{i^{*}}, U_{i_{*}}$, and $U_{i^{s e l}}$ are the utilities of the best, worst and chosen (by SMAA) alternatives according to the simulated 'true' rank order. In the current paper, we have elected to use rank-based measures as the analytical results reported in Section 5 are much easier to obtain with these than with utility losses, which depend both on what rank an alternative obtains and the distribution of utilities over alternatives. In Section 4.1 we report utility loss for preliminary simulations and show that it is strongly correlated to the probability that MAUT and SMAA select the same alternative.

\subsection{Parameter values used}

In simulation studies such as this it is usually necessary to include only a small number of possible values for each simulation parameters. This keeps the total computation time and model complexity within reasonable limits, and should not be interpreted as indicating that these are the only plausible values or even capture the full range of possible variation.

The effect of problem size is investigated using $I=9$ or 19 alternatives and $J=6$ or 12 attributes. The four combinations allow for an independent investigation of alternatives and attributes in decision environments located between 'fairly small' and 'moderate' in size. Similar values have been used in Barron and Barrett (1996) and Salo and Hämäläinen (2001).

Parameter values for the variance and skewness parameters $\left(\sigma_{i j}\right.$ and $\xi_{i j}$ respectively) were chosen on the basis of trial-and-error experimentation. The variance parameter takes on either "low" $\left(\sigma_{i j} \sim U n[0.02,0.04]\right)$ or "high" $\left(\sigma_{i j} \sim U n[0.08,0.1]\right)$ values. For the symmetric case and prior to standardization, a $6 \sigma$ range will cover approximately $25 \%$ and $45 \%$ of the full range of possible attribute evaluations in the 'low' and 'high' variability conditions respectively. After standardisation these ranges are somewhat larger; pre-simulations indicated coverage of $40-50 \%$ and $65-70 \%$ respectively. This means that even in the 'low' variability condition there is still a considerable amount of uncertainty in the attribute evaluations. The skewness of the attribute evaluations is considered to be either zero (in 
which case the gamma distribution is equivalent to a Gaussian distribution), or a moderate positive value randomly drawn from a uniform distribution $\xi_{i j} \sim U n[0.5,1] \forall i, j$. The assessment error parameter $\epsilon$ is key and so takes on one of three values : $0 \%, 10 \%$, and $20 \%$.

Parameter values for $\tau_{j}$ and $\lambda_{j}$ are combined to give preferences that are convex $\left(\tau_{j}=\right.$ $\left.0.8, \lambda_{j}=0.2\right)$, concave $\left(\tau_{j}=0.2, \lambda_{j}=0.8\right)$, or linear $\left(\tau_{j}=\lambda_{j}\right)$. Note that $\tau_{j}$ and $\lambda_{j}$ are not varied independently. A single parameter $\zeta$ controls preferences for risk in the utility function defined over scalar value, and thus maps global values into utilities using equation (1): we use $\zeta=2$ (constant risk aversion with respect to value), $\zeta=0$ (risk neutral), and $\zeta=-2$ (constant risk proneness).

SMAA models are based upon the best $R=\{1,3,5\}$ ranks. The effect of including partial preference information about the weights into the SMAA process is investigated as part of an initial set of simulations using $I=19$ alternatives, $J=12$ attributes, and $\rho \in\{0,1 / 11,3 / 11,5 / 11,8 / 11,1\}$, with 500 runs for each value of $\rho$. We also simulated an additional 'full-information' case where the cardinal values of weights are known precisely. Other variables were held constant at high $\sigma_{i j}$ and $\xi_{i j}, \epsilon=0$, mostly convex utility (low $\tau_{i j}$, high $\lambda_{i j}$ ), and $\zeta=0$. On the basis of these results, reported in Section 4.1, an "intermediate" value of $\rho=0.5$ was selected for use in the full simulation study.

We used a full factorial design - with 16 parameter combinations for problem context, 3 for assessment error, and 6 for shape of utility function there are a total of 288 simulation parameter combinations. We perform 100 simulation runs for each combination of parameters, and each simulation run uses 10000 random weight vectors to generate the SMAA results. Simulations were run using $\mathrm{R}$ version 3.1.3 and used packages hitandrun version 0.5 and smaa version 0.2-3 (van Valkenhoef, 2014).

\section{Results}

\subsection{Weight restrictions}

Figure 2 illustrates the relationship between the amount of weight information provided and various measures of model accuracy. In this and other plots, error bars indicate $95 \%$ confidence intervals around the mean values. If uncertainty about attribute evaluations is fully specified but no weight information is provided at all, the probability that a SMAA model selects the same alternative as MAUT is 0.15 , and the probability of that alternative appearing in the top three or five ranks (out of 19) of the SMAA rank order is 0.33 , and 
0.50 respectively. If ordinal weight information is fully specified in decreasing order of importance these probabilities increase to $0.77,0.97$, and 0.99 respectively.

Marginal improvements in model accuracy diminish with the importance of the attribute for which ordinal information is specified. The greatest improvement in model accuracy comes from providing ordinal information about a few of the most important attributes. In this case, information about attributes in the lower half of the importance rank order produces very little additional improvement in model accuracy. Marginal returns diminish, but more slowly, if information is provided on the least important attributes first.

Figure 2 also shows that the way in which uncertainty about attribute evaluation is specified also has a strong effect on model accuracy, particularly on the probability of the SMAA and MAUT models selecting the same alternative as best, and particularly when some ordinal information about attribute importance is provided.

Finally, Figure 2 shows that our four outcome measures are strongly correlated, responding in much the same way to how the uncertainty about attribute evaluations is represented, and to the provision of ordinal weight information. Correlations range between 0.41 (between the probability of selecting the preferred MAUT alternative in the top and top 5 positions of the SMAA rank order) and -0.58 (between utility loss and the probability that the SMAA model selects the same alternative as MAUT).

On the basis of this preliminary investigation, we fix $\rho$ at an intermediate value of 0.5 and in the remainder of this section explore the effect that other parameters have in more detail. Figure 3 reports mean model accuracy for different uncertainty models using $R$-best-ranks in the computation of the acceptability index, under various error conditions and values of $R$. Results have been averaged over the remaining simulation parameters.

We report three results here. Firstly, errors in the assessment of uncertainty can seriously compromise model accuracy. The greatest deterioration, as a result of assessment errors, occurs in the probability that the SMAA model selects the same alternative as MAUT (relative to the probability of shortlisting this alternative), and in SMAA models representing uncertainty with probability distributions. Some caution is needed in interpreting this latter result, since assessment errors are not directly comparable across uncertainty types. It is not clear, for example, whether it is cognitively "easier" to make a $10 \%$ error when assessing parameters of probability distributions or when assessing expected values. 
Figure 2: Mean model accuracy as a function of the degree of imprecision in the weight space. Note ' $1+\omega$ ' refers to the full-information case where the cardinal values of weights are known precisely. 
Secondly, models using simplified representations of uncertainty, particularly quantiles, can return very similar results to models using the full probability distribution. Models using explicit risk measures, however, performed poorly on average. The same finding was reported in the context of utility/value function approaches with known weights in Durbach and Stewart (2012); the current result extends this to the SMAA context.

Finally, in the absence of assessment error model accuracy is almost always highest when only the first rank is used to construct the SMAA acceptability index. The sole exception is the risk-based model - the probability of the preferred MAUT appearing in a shortlist is increased if the acceptability index is composed of the same number of ranks as the shortlist. However, when assessment errors are present, the same effect - the probability of the preferred MAUT alternative appearing in a shortlist is increased if the acceptability index is composed of the same number of ranks as the shortlist - is observed in other models too.

Figure 4 shows two results related to the size of the decision problem considered. Model accuracy deteriorates as the number of alternatives increases, but improves as the number of attribute increases. This occurs for all of the simulated models, which to rough approximation experience the same magnitude of improvements and deteriorations.

Figure 5 shows that in general skewness and variability in attribute evaluations lead to lower model accuracy. Models using simplified representations of uncertainty (those using quantiles, expected values, or variances) suffer far more from increases in variability or skewness of attribute evaluations than do SMAA models using full probability distributions. In fact, increasing attribute variability does not significantly change the accuracy of the SMAA models using full probability distributions. The net effect is that quantile models become less attractive as distributions become more variable and skew.

Figure 6 shows mean utility losses for different types of marginal value functions i.e. combinations of $(\tau, \lambda)$, and risk preference parameter $\zeta$. Model accuracy is highest for all models when marginal value functions are convex, deteriorating as these become linear and then concave. The effect of multi-attribute risk aversion $\zeta$ is substantially less than that of the shape of the marginal value functions, and contrasts are for the most part nonsignificant. The effect of risk preferences over value are only significant when marginal value functions are convex, shown in the right-hand plot in Figure 6, in which case accuracy is highest when decision makers are multivariate risk neutral. 


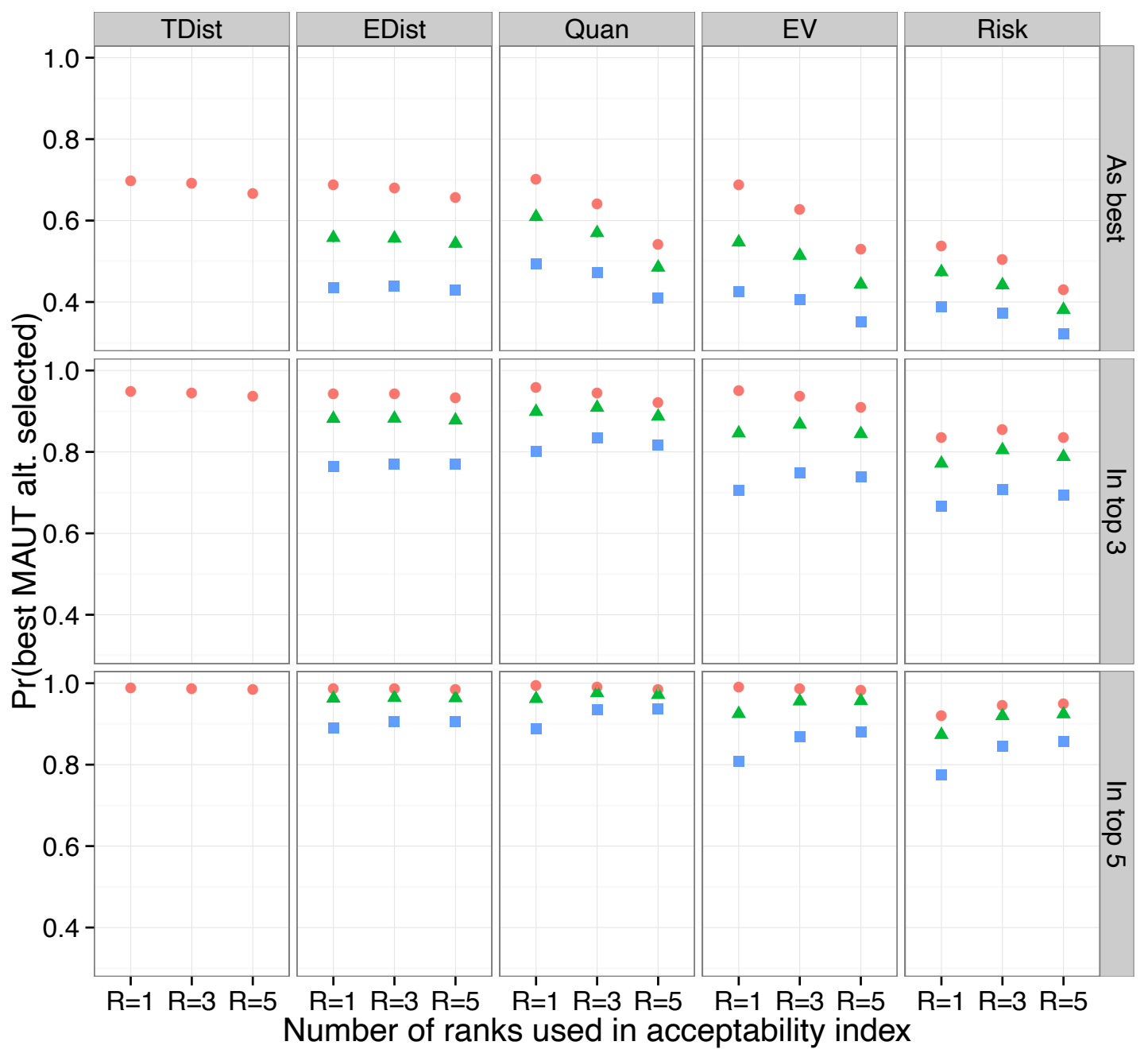

error - none $-10 \%-20 \%$

Figure 3: Mean model accuracy as a function of assessment error levels and number of ranks used in the construction of acceptability indices. 


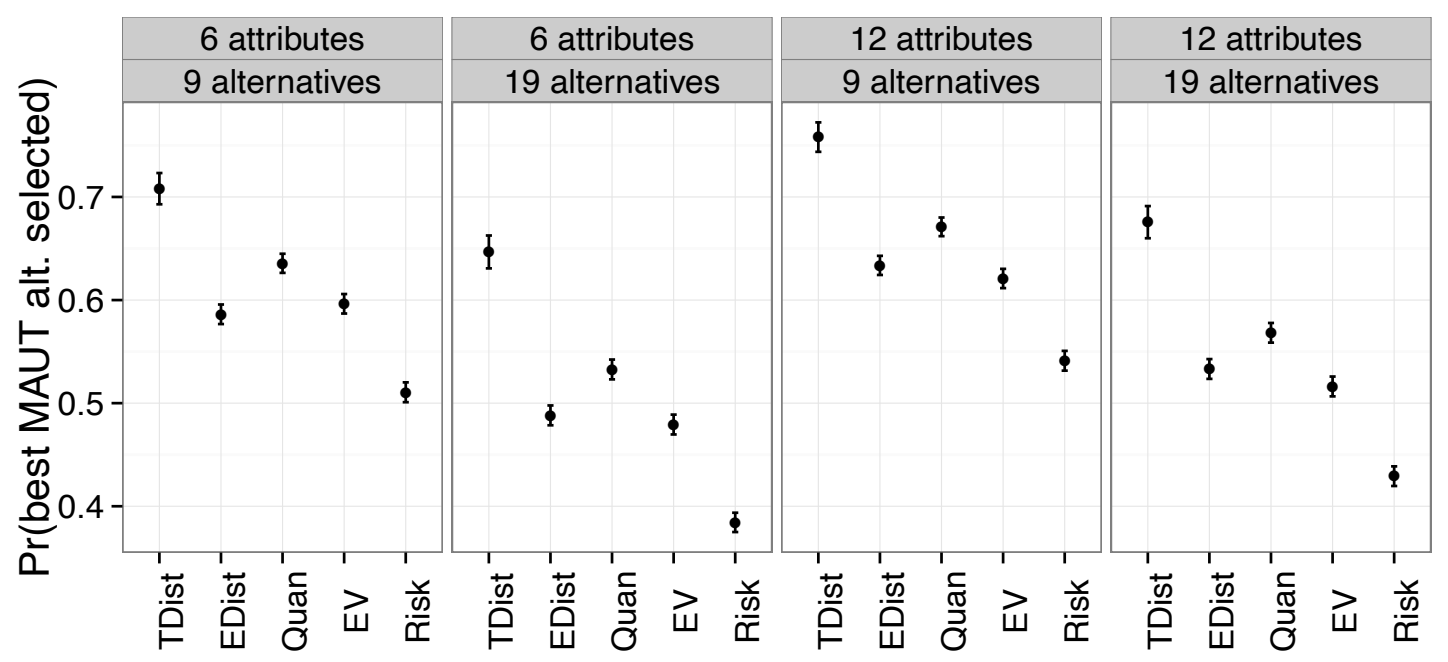

Figure 4: Mean model accuracy as a function of the number of alternatives and attributes present in the decision problem.

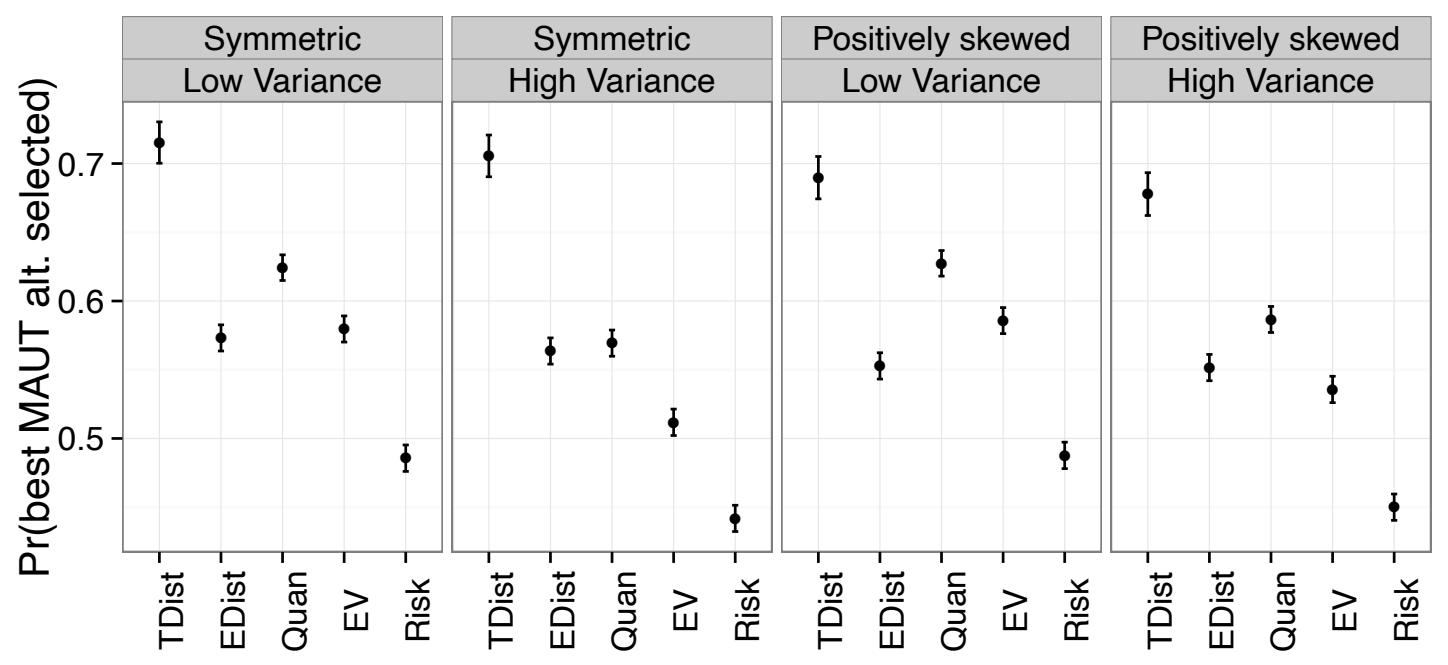

Figure 5: Mean model accuracy as a function of the variance and skewness of distributions governing attribute evaluations. 


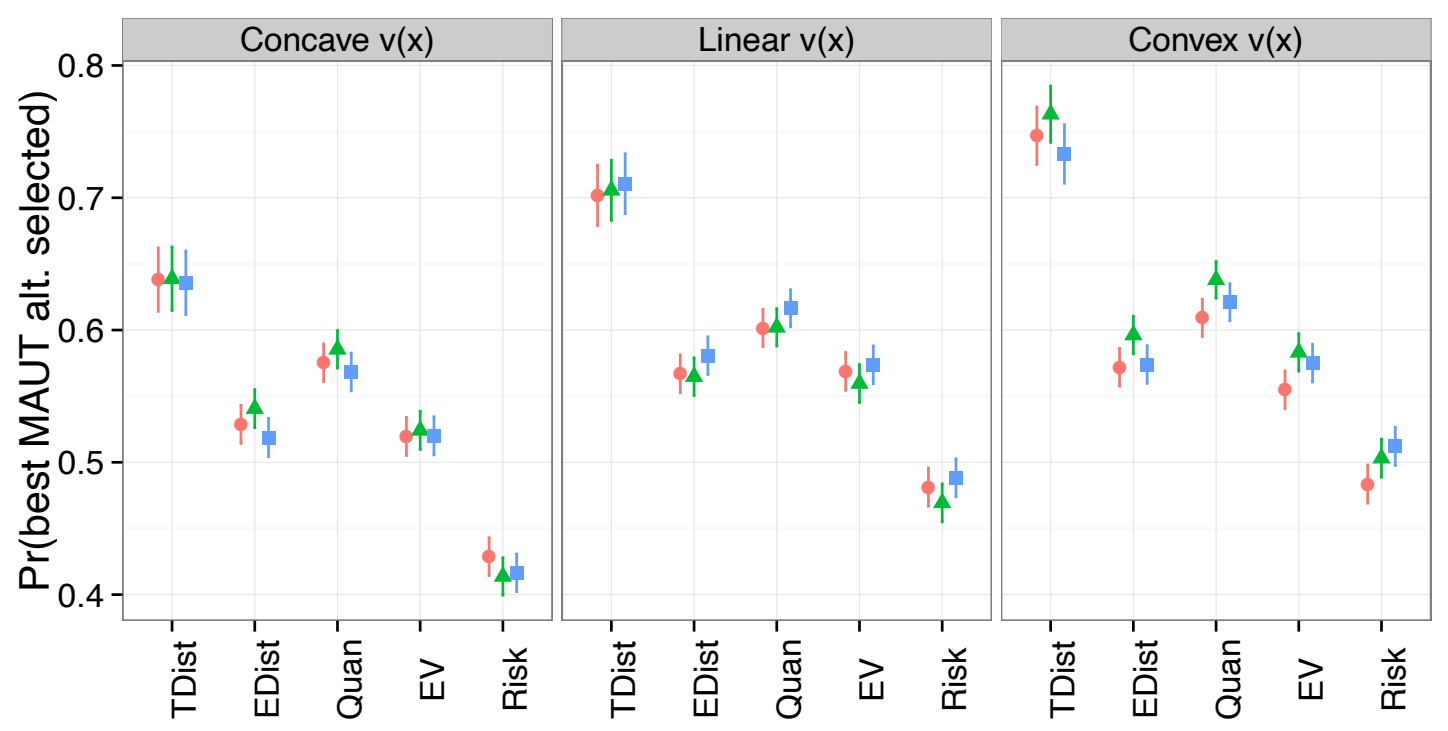

Multiv.Risk $\rightarrow$ Constant risk prone $\rightarrow$ Risk neutral $\rightarrow-$ Constant risk averse

Figure 6: Mean model accuracy as a function of DM preferences, as indicated by the shape of marginal value functions and risk preferences defined over global value.

\section{Discussion}

Since SMAA always selects the alternative with the largest acceptability index, the probability that the SMAA model selects the same alternative as MAUT is equivalent to computing the probability that the decision maker's true preferences lie inside the largest favourable weight space. This is precisely what is computed by the largest rank-1 acceptability index. We therefore examine distributional properties of the random variable $B_{(k)}^{1}$ denoting the $k$-th largest rank-1 acceptability index, and in particular the expected value of the largest rank-1 acceptability index $E\left[B_{(1)}^{1}\right]$. Unfortunately deriving general analytical results for $B_{(k)}^{1}$ is difficult, so that we first consider an artificially simple case where exact results can be obtained, and then extrapolate from these using heuristic arguments.

\subsection{Model accuracy deteriorates as the number of alternatives increases}

Suppose we have two attributes and $I$ non-dominated alternatives, with known attribute evaluations and linear value functions. The weight space in this case consists of the line segment $[0,1]$ i.e. the 1 -simplex, from which $w_{1}$ and $w_{2}$ are obtained directly. The allocation of parts of the weight space to different alternatives, and hence the computation of acceptability indices, is analogous to the problem of breaking this line segment at 
random into $I$ pieces, and randomly allocating each piece to an alternative. An alternative $a_{i}$ is then selected with probability proportional to the length of the line segment allocated to it.

The distribution of the length of the $k$-th largest piece of the broken line segment is a well-studied problem in applied probability (where the line segment is often thought of as a "stick" of unit length) and is given by (e.g. Holst, 1980)

$$
P\left(B_{(k)}^{1} \leq x\right)=\sum_{m=0}^{k-1}\left(\begin{array}{c}
I \\
m
\end{array}\right) \sum_{n=0}^{I-m}(-1)^{n}\left(\begin{array}{c}
I-m \\
n
\end{array}\right)(1-(m+n) x)_{+}^{I-1}
$$

where $x_{+}=\max (x, 0)$. Under the assumptions of the broken stick model ${ }^{2}$, this then is also the distribution of $k$-th largest rank-1 acceptability index, from which one obtains the expected $k$-th largest rank-1 acceptability index as

$$
E\left[B_{(k)}^{1}\right]=1 / I \sum_{m=0}^{I-k} 1 /(I-m)
$$

The largest rank-1 acceptability index, and hence the probability that the SMAA model selects the same alternative as MAUT, thus has expected value $E\left[B_{(1)}^{1}\right]=(1 / I) \sum_{m=0}^{I-1} 1 /(I-$ 1) $\sim \log (I) / I$, which decreases with the number of alternatives $I$.

\subsection{Model accuracy improves as the number of attributes increases}

With $J=2$ attributes the pieces of the broken stick directly give the probability of selecting alternative $a_{i}$. Extending this to $J>2$ attributes requires $J-1$ sticks, each of which is broken at random into $I$ pieces, with pieces allocated at random to alternatives. Alternative $a_{i}$ is then selected with probability proportional to the size of the hypervolume formed by the pieces allocated to it, computed as the product of the lengths of its $J-1$ pieces. This construction effectively assumes that the length of piece $i$ of stick $j$ represents the marginal likelihood of selecting alternative $a_{i}$ after integrating over all dimensions of the weight space other than the one representing attribute $c_{j}$. Obtaining distributional properties of order statistics over these product terms is difficult, but a heuristic argument can be used to show that the expected value of the largest acceptability index increases in the number of attributes used.

Since each line segment is assumed independent and identically distributed, the expected product of the $k$-th longest piece of each line segment is given by $\prod_{j=1}^{J-1} 1 / I \sum_{m=0}^{I-k} 1 /(I-$

\footnotetext{
${ }^{2}$ The assumptions of the broken stick model - uniformly and independently distributed breakpoints may be less likely to hold in SMAA where there may be interactions between the regions of optimality for different alternatives linked to, for example, correlations in their performance.
} 
$m) \sim(\log (I-k+1) / I)^{J-1}$. This expected product decreases as the number of attributes $J$ increases, but decreases much faster for larger $k$ i.e. for products of small pieces than for products of large pieces. Thus, when renormalizing the hypervolumes, the net effect is that the expected value of the largest acceptability index increases with $J$. A simple numerical experiment was used to confirm that this remains true when products are formed from arbitrary pieces of broken line segments, as described above. With two alternatives and two (ten, thirty) attributes $E\left[B_{(1)}^{1}\right]=0.75(0.83,0.89)$.

The preceding argument is based upon a problem context in which weights are entirely unknown. In our simulations ordinal rank information was provided on half the attribute weights, and thus an alternate explanation for the increase in $J$ is that we are providing absolutely more weight information. This explanation was ruled out by observing the same effect in additional simulations run with no weight information $(\rho=0)$.

\subsection{Model accuracy and ordinal weight importance information}

In the absence of any weight importance information the probability the decision maker's true preferences lie inside the largest favourable weight space is simply the proportion of the total feasible weight space occupied by the largest favourable weight space. Restricting the weight space increases this probability whenever it is done in such a way that the total feasible weight space $W^{\prime}$ shrinks by a greater proportion than the largest favourable weight space, provided that the feasible weight space still contains the decision maker's true preferences ${ }^{3}$. In a uniformly distributed weight space, each ordinal constraint of the form $w_{j}>w_{k}$ eliminates half of the remaining weight space from consideration. Thus the first constraint eliminates half the weight space, the second a quarter, and so on. Knowing the importance ranking of one attribute introduces $J-1$ ordinal constraints and thus eliminates $100\left(1-0.5^{J-1}\right) \%$ of the feasible weight space. Knowing the importance ranking of a second attribute introduces $J-2$ ordinal constraints and thus eliminates a fraction $1-0.5^{J-2}$ of the remaining feasible weight space. For example, for $J=5$ knowing the importance rank of one alternative eliminates 15/16 of the weight space, while knowing the rank of a second alternative eliminates $7 / 8$ of the remaining $1 / 16$, that is just $7 / 128$ of the original weight space. Thus it is unsurprising that marginal improvements in accuracy diminish as more attribute importance rankings become known, as less and less of the

\footnotetext{
${ }^{3}$ Once restrictions to the weight space are applied we must take into account the possibility that the restrictions incorrectly exclude the decision makers true preferences. In this case the probability that the SMAA and MAUT models select the same alternative is $E\left[B_{(1)}^{1}\right] P\left(\omega \in W^{\prime}\right)$ where $\omega$ is the decision maker's true weights.
} 
weight space is removed at each step. In fact, given the argument above it is interesting that marginal improvements diminish as slowly as they do. The reason for this must lie in each weight restriction eliminating only slightly less of the largest favourable weight space, as a proportion of its original volume, than is true of the total feasible weight space.

To the extent that weight restrictions increase $b_{(1)}^{1}$, they do this by reducing the rank-1 acceptability indices of other alternatives. Since weights express trade-offs, alternatives that do well on an unimportant attribute must, other things being equal, do much better on this attribute to have any chance of being selected. Information which sorts between relatively unimportant attributes therefore, on average, eliminates relatively fewer alternatives - or equivalently, reduces the acceptability indices of other alternatives less - than information sorting between more important attributes.

\subsection{Model accuracy and value functions}

Model accuracy is highest when marginal value functions are convex and multivariate risk attitude is neutral. As the SMAA models all make the simplifying assumption that that the DM is multivariate risk neutral, the latter result is to be expected and can be interpreted as an artifact of the simulation structure. More notable is the relative insensitivity of results to multivariate risk aversion, although it may be reasonably argued that this is because we varied the risk aversion parameter $\zeta \in[-2,2]$ too narrowly.

A convincing explanation for the effect of the shape of the marginal value functions is more elusive. We can only conjecture here that convex marginal value functions help differentiate between good alternatives. For convex functions $v_{j}(x)-v_{j}(x-d)>v_{j}(y)-$ $v_{j}(y-d)$ iff $x>y$, so that equal size differences in performances on the attribute scale translate into larger value difference the better the performance levels are. Thus, the difference between the largest and second-largest acceptability indices, $b_{(1)}^{1}-b_{(2)}^{1}$, is likely to be larger when marginal value functions are convex.

\subsection{Shortlists are more robust to assessment errors than choices}

Errors in the assessment of the uncertainty of attribute evaluations introduce errors in the estimation of acceptability indices. If these estimation errors are sufficiently large the alternative with the largest (true) rank-1 acceptability index $b_{(1)}^{1}$ may not appear first in the SMAA rank order. If this occurs it is clear that it is more likely that alternative $a_{(1)}$ appears, for example, in the second rank than in the worst rank, or more generally in rank $k$ than in rank $m>k$. Thus, assessment errors affect the probability of including 
the preferred MAUT alternative in a shortlist less than the probability of selecting this alternative as best.

It may be possible to derive analytical results on the necessary errors from the expression for $P\left(B_{(k)}^{1} \leq x\right)$ in (9) by assuming, for example, that a rank reversal occurs when $B_{(1)}^{1}-B_{(2)}^{1} \leq \delta$, where $\delta$ is a random error in the estimation of acceptability indices. Given the difficulty of deriving $P\left(B_{(k)}^{1}\right)$ for more complex decision problems, and of deriving real-world recommendations from the error term $\delta$, we do not pursue this further here.

\section{Conclusion}

Our simulation evaluates the ability of acceptability indices derived from SMAA models to approximate MAUT, and in particular SMAA models that use simplified formats for representing uncertainty in the attribute evaluations. The previous section elaborated on possible reasons for some of the more important of the simulation results.

In this section we summarize the contribution of the paper and conclude with some tentative recommendations for practice, including when different SMAA models might be more or less useful. These recommendations should be interpreted with caution though, since they require a judgment about what level of performance (e.g. in terms of the probability of selecting the same alternative as MAUT) is acceptable, which is necessarily problem dependent. Furthermore, any recommendations regarding responses to changes in a problem input (e.g. more attributes) that are derived from simulation results are heavily dependent on what values assumed for other variables, and others may reasonably disagree with ours. Thus, our recommendations only hold with these two caveats in mind.

Our main conclusions regarding the implementation of SMAA to support low-involvement decision making are as follows:

1. Acceptability indices obtained from SMAA models can be expected to return reasonable approximations to the full MAUT model in certain contexts and for certain problematiques (see below).

2. The accuracy of SMAA models improves as more ordinal weight importance information is provided, but

(a) marginal improvements diminish rather quickly with the number of the attributes for which importance rankings are specified, and 
(b) the rate at which marginal returns diminish is greatest when the most important attributes are specified first, and

(c) if no weight information is provided at all, the average accuracy of all SMAA models is poor.

3. It is not essential that the SMAA model uses probability distributions to represent attribute uncertainty. Good results can also be obtained using simplified uncertainty formats, particularly quantiles.

4. Errors in the assessment of the uncertainty of attribute evaluations affect the probability of including the preferred MAUT alternative in a shortlist less than the probability of selecting this alternative as best.

5. The accuracy of the SMAA models deteriorates as attribute evaluations become more skew and, for those models using simplified representations of uncertainty, more variable. Quantile models thus become less attractive in highly variable problem contexts.

6. The accuracy of all SMAA models deteriorates as the number of alternatives increases, but improves as the number of attributes increases.

Taken together, our results suggest some role for SMAA in low-involvement decision making. It seems that, with limited preference information and summarised representations of uncertain attribute evaluations, the SMAA acceptability index can be used to rank order alternatives and select one or a small number which, on average, perform relatively well. However under the wrong kinds of conditions performance can be poor and thus avoiding these conditions, or responding adequately to them, is important part of providing good decision support. To this end our recommendations for practice are:

1. Deciding between alternatives based on SMAA acceptability indices without some restriction of the weight space cannot be recommended. There is at most a $50 \%$ chance that the preferred MAUT alternative appears in the top five out of 19 alternatives, even when no assessment errors are made.

2. The SMAA acceptability index can be justifiably used for deciding between alternatives if either the weight space is restricted or indices are used to shortlist rather than choose between alternatives. Conservatively, the most important $25 \%$ of the 
attribute set should be identified and ordered, and a shortlist of $15 \%$ or more of the alternatives set extracted.

3. The acceptability index should always use the same number of ranks as the size of the constructed shortlist. These are more robust to assessment errors and offer only slightly worse performance in error-free environments.

4. Uncertainty about attribute evaluations should be assessed using either the full probability distributions, or quantiles. Expected values and variance measures are more sensitive to assessment errors and worse on average than other models, even if correctly assessed. These are thus not recommended.

5. Errors in the assessment of uncertain attribute evaluations cause substantial deteriorations in model quality. If assessment errors are expected e.g. because of limited time or experience, the size of the constructed shortlist should be extended (speculatively, on the basis of simulation results, perhaps doubled).

6. The approach outlined above is suitable for large attribute sets and in fact will return higher-quality shortlists as the number of attributes increases. Alternatively, the DM may be able to specify importance information for a slightly smaller proportion of the attribute set or use a smaller shortlist of alternatives.

In a simulation study such as this we are unable to assess a number of practical issues. For example: whether the reduced accuracy is "acceptable" for DMs; whether the tradeoff between accuracy and the time saved on weight elicitation is felt to be worthwhile; or whether the limited weight information that must be assessed for the SMAA models is felt to still be too onerous a task. More broadly, while efficient sampling procedures are now available for a wide range of weight constraints in MCDA, the practical assessment of these constraints remains unresolved. For example, are DMs able to reliably restrict the weight space, in the sense of offering constraints that do not exclude their true preferences? What types of constraints are DMs more comfortable providing information on? What are the best procedures for aiding this process? Investigating these important practical issues offers a clear path for future research. 


\section{References}

B.S. Ahn and H. Park. Establishing dominance between strategies with interval judgments of state probabilities. Omega, 49:53-59, 2014.

S. Angilella, S. Corrente, and S. Greco. Stochastic multiobjective acceptability analysis for the choquet integral preference model and the scale construction problem. European Journal of Operational Research, 240(1):172-182, 2015.

F.H. Barron and B.E. Barrett. Decision quality using ranked attribute weights. Management Science, 42(11):1515-1523, 1996.

J. Butler, J. Jia, and J. Dyer. Simulation techniques for the sensitivity of multi-criteria decision models. European Journal of Operational Research, 103:531-546, 1997.

I. Durbach. A simulation-based test of stochastic multicriteria acceptability analysis using achievement functions. European Journal of Operational Research, 170:923-934, 2006.

I. Durbach and S. Davis. Decision support for selecting a shortlist of electricity-saving options: A modified SMAA approach. ORiON, 28(2):99-116, 2013.

I. Durbach, R. Lahdelma, and P. Salminen. The analytic hierarchy process with stochastic judgements. European Journal of Operational Research, 238(2):552-559, 2014.

I.N. Durbach and T.J. Stewart. Using expected values to simplify decision making under uncertainty. Omega, 37(2):312-330, 2009.

I.N. Durbach and T.J. Stewart. A comparison of simplified value function approaches for treating uncertainty in multi-criteria decision analysis. Omega, 40:456-464, 2012.

J. Hokkanen, R. Lahdelma, K. Miettinen, and P. Salminen. Determining the implementation order of a general plan by using a multicriteria method. Journal of Multi-Criteria Decision Analysis, 7(5):273-284, 1998.

L. Holst. On the lengths of the pieces of a stick broken at random. Journal of Applied Probability, pages 623-634, 1980.

A. Jessop. Imp: A decision aid for multiattribute evaluation using imprecise weight estimates. Omega, 49:18-29, 2014. 
M. Kadziński, T. Tervonen, and J.R. Figueira. Robust multi-criteria sorting with the outranking preference model and characteristic profiles. Omega, 55:126-140, 2015.

D.L. Keefer and S.E. Bodily. Three-Point Approximations for Continuous Random Variables. Management Science, 29(5):595-609, May 1983.

R. Keeney and H. Raiffa. Decisions with Multiple Objectives: Preferences and Value Tradeoffs. John Wiley \& Sons, New York, 1976.

C. Kirkwood. Estimating the impact of uncertainty on deterministic multiattribute evaluation. Management Science, 38(6):819-826, 1992.

R. Lahdelma and P. Salminen. SMAA-2: stochastic multi-criteria acceptability analysis for group decision making. Operations Research, 49(3):444-454, 2001.

R. Lahdelma and P. Salminen. Prospect theory and stochastic multicriteria acceptability analysis (SMAA). Omega, 37(5):961-971, 2009.

R. Lahdelma and P. Salminen. Stochastic multicriteria acceptability analysis (SMAA). In S. Greco, M. Ehrgott, and J.R. Figueira, editors, Trends in Multiple Criteria Decision Analysis, chapter 10. Springer, 2012.

R. Lahdelma, J. Hokkanen, and P. Salminen. SMAA - Stochastic multiobjective acceptability analysis. European Journal of Operational Research, 106:137-143, 1998.

R. Lahdelma, K. Miettinen, and P. Salminen. Reference point approach for multiple decision makers. European Journal of Operational Research, 164(3):785-791, 2005.

R Core Team. R: A Language and Environment for Statistical Computing. R Foundation for Statistical Computing, Vienna, Austria, 2014. URL http://www.R-project.org/.

A.A. Salo and R.P. Hämäläinen. Preference ratios in multiattribute evaluation (PRIME) - elicitation and decision procedures under incomplete information. IEEE Transactions on Systems, Man, and Cybernetics, Part A, 31(6):533-545, 2001.

T.J. Stewart. Robustness of additive value function methods in MCDM. Journal of Multi-criteria Decision Analysis, 5:301-309, 1996.

T. Tervonen and J.R. Figueira. A survey on stochastic multicriteria acceptability analysis methods. Journal of Multi-Criteria Decision Analysis, 15(1-2):1-14, 2008. 
$\mathrm{T}$ Tervonen and R Lahdelma. Implementing stochastic multicriteria acceptability analysis. European Journal of Operational Research, 178(3):500-513, 2007.

T. Tervonen, G. van Valkenhoef, N. Baştürk, and D. Postmus. Hit-and-run enables efficient weight generation for simulation-based multiple criteria decision analysis. European Journal of Operational Research, 224(3):552-559, 2013.

G. van Valkenhoef, T. Tervonen, and D. Postmus. Notes on hit-and-run enables efficient weight generation for simulation-based multiple criteria decision analysis. European Journal of Operational Research, 239(3):865-867, 2014.

G. van Valkenhoef. smaa: Stochastic Multi-criteria Acceptability Analysis, 2014. URL http://CRAN.R-project.org/package=smaa. R package version 0.2-3.

G. van Valkenhoef and T. Tervonen. hitandrun: "Hit and Run" and "Shake and Bake" for Sampling Uniformly From Convex Shapes, 2015. URL http://CRAN.R-project.org/package=hitandrun. R package version 0.5. 